\title{
Article
}

\section{Solar interacting protons versus interplanetary protons in the core plus halo model of diffusive shock acceleration and stochastic re- acceleration}

Kocharov, L, Laitinen, Timo Lauri mikael, Vainio, R, Afanasiev, A, Mursula, M and Ryan, JM

Available at http://clok.uclan.ac.uk/12029/

Kocharov, L, Laitinen, Timo Lauri mikael ORCID: 0000-0002-7719-7783, Vainio, $R$, Afanasiev, A, Mursula, M and Ryan, JM (2015) Solar interacting protons versus interplanetary protons in the core plus halo model of diffusive shock acceleration and stochastic re-acceleration. The Astrophysical Journal, 806 (80). ISSN 0004-637X

It is advisable to refer to the publisher's version if you intend to cite from the work. http://dx.doi.org/10.1088/0004-637X/806/1/80

For more information about UCLan's research in this area go to http://www.uclan.ac.uk/researchgroups/ and search for <name of research Group>.

For information about Research generally at UCLan please go to http://www.uclan.ac.uk/research/

All outputs in CLoK are protected by Intellectual Property Rights law, including Copyright law. Copyright, IPR and Moral Rights for the works on this site are retained by the individual authors and/or other copyright owners. Terms and conditions for use of this material are defined in the policies page. 


\title{
SOLAR INTERACTING PROTONS VERSUS INTERPLANETARY PROTONS IN THE CORE PLUS HALO MODEL OF DIFFUSIVE SHOCK ACCELERATION AND STOCHASTIC RE-ACCELERATION
}

\author{
L. Kocharov ${ }^{1}$, T. Laitinen ${ }^{2}$, R. Vainio ${ }^{3}$, A. Afanasiev ${ }^{3}$, K. Mursula ${ }^{4}$, and J. M. Ryan ${ }^{5}$ \\ ${ }^{1}$ Sodankylä Geophysical Observatory (Oulu Unit), P.O. Box 3000, University of Oulu, FI-90014 Oulu, Finland \\ 2 Jeremiah Horrocks Institute, University of Central Lancashire, Preston PR1 2HE, UK \\ ${ }^{3}$ Department of Physics and Astronomy, University of Turku, FI-20014 Turku, Finland \\ ${ }^{4}$ Department of Physics, University of Oulu, FI-90014 Oulu, Finland \\ ${ }^{5}$ Space Science Center, Institute for the Study of Earth, Oceans and Space, University of New Hampshire, Durham, NH 03824, USA \\ Received 2014 November 21; accepted 2015 April 20; published 2015 June 9
}

\begin{abstract}
With the first observations of solar $\gamma$-rays from the decay of pions, the relationship of protons producing ground level enhancements (GLEs) on the Earth to those of similar energies producing the $\gamma$-rays on the Sun has been debated. These two populations may be either independent and simply coincident in large flares, or they may be, in fact, the same population stemming from a single accelerating agent and jointly distributed at the Sun and also in space. Assuming the latter, we model a scenario in which particles are accelerated near the Sun in a shock wave with a fraction transported back to the solar surface to radiate, while the remainder is detected at Earth in the form of a GLE. Interplanetary ions versus ions interacting at the Sun are studied for a spherical shock wave propagating in a radial magnetic field through a highly turbulent radial ray (the acceleration core) and surrounding weakly turbulent sector in which the accelerated particles can propagate toward or away from the Sun. The model presented here accounts for both the first-order Fermi acceleration at the shock front and the second-order, stochastic re-acceleration by the turbulence enhanced behind the shock. We find that the re-acceleration is important in generating the $\gamma$-radiation and we also find that up to $10 \%$ of the particle population can find its way to the Sun as compared to particles escaping to the interplanetary space.
\end{abstract}

Key words: acceleration of particles - plasmas - shock waves - Sun: coronal mass ejections (CMEs) Sun: particle emission - turbulence

\section{INTRODUCTION}

Major solar eruptions, i.e., solar flares and coronal mass ejections (CMEs), can accelerate ions up to several $\mathrm{GeV}$, which is evident from observations of $\gamma$-ray lines produced by high-energy ions colliding in the solar atmosphere and from observations of accelerated ions arriving at $1 \mathrm{AU}$. However, the relationship of the solar interacting particles to the solar energetic particles (SEPs) in space has long been a matter of controversy (e.g., Lin 2005; McCracken et al. 2008). They may originate either from two different sources or from a single source. Hybrid scenarios, which would comprise both common and separate sources for interacting particles and SEPs, could not be ruled out either, e.g., the first source ("flare source") for only interacting particles, the second source ("coronal source") contributing to both particle populations, and the third source ("interplanetary source") only for SEPs (Kocharov et al. 1996). The number ratio of ions escaping into the interplanetary medium to ions precipitating into the solar chromosphere, $N_{\uparrow} / N_{\downarrow}$, may vary from one solar $\gamma$-ray flare to another by a few orders of magnitude, with larger values associated with gradual flares as compared to impulsive ones (Murphy \& Ramaty 1984; Hua \& Lingenfelter 1987; Ramaty et al. 1993).

In impulsive flares, most of the high-energy ions are accelerated and trapped in coronal magnetic loops. Correspondingly, the fraction of the $>30 \mathrm{MeV}$ protons that escape into the interplanetary medium is small, $N_{\uparrow}(>30 \mathrm{MeV}) \leqslant 0.1 N_{\downarrow}$ $(>30 \mathrm{MeV})$, while this is not the case in gradual flares (Figure 17 and Table 2 by Hua \& Lingenfelter 1987). The most modern $\gamma$-ray instrument, the Large Area Telescope onboard the Fermi Gamma-ray Space Telescope spacecraft, is much more sensitive than the instruments were thirty years ago and thus allows accurate measurements of the prolonged $\gamma$-ray emissions with energy $>100 \mathrm{MeV}$ associated with gradual solar events (Ackermann et al. 2014; Ajello 2014). Nowadays, even $\gamma$-ray emission produced by a relatively small number of interacting particles, $N_{\downarrow} \ll N_{\uparrow}$, can be observed.

Major (gradual) SEP events in the energy range $\sim 1 \mathrm{MeV}-$ $100 \mathrm{MeV}$ are associated with CME-driven shocks in solar corona and solar wind (Lee \& Ryan 1986; Lee et al. 2012, and references therein). Even relativistic protons causing the ground level enhancements (GLEs) may be produced by the shocks (Debrunner et al. 1993), within $5 R_{\odot}$ from the Sun (Kahler 1994; Aschwanden 2012). There are also indications that low-energy ions ("seed ions") for the GLE-producing shock acceleration may be provided by the flare-type process associated with the preceding CME (Mewaldt et al. 2012). In this paper, we will consider a model of the high-energy $(\gtrsim 100 \mathrm{MeV})$ proton acceleration by the CME shock at (1.5-6) $R_{\odot}$. Thus, our modeling is focused on the major (gradual) solar high-energy particle events and addresses the question whether CME-driven shocks can produce both interplanetary and interacting particles after the impulsive phase of the associated solar flare.

\section{THEORETICAL MOTIVATION}

Shock waves in astrophysical plasmas can efficiently accelerate charged particles via the diffusive shock acceleration mechanism, if the particle scattering near the shock is frequent (e.g., Toptygin 1985). The scattering can be provided either by external (pre-existing) turbulence (Axford et al. 1977; Krymsky 1977; Blandford \& Ostriker 1978) or by the accelerated particles themselves via the resonant waves excited 


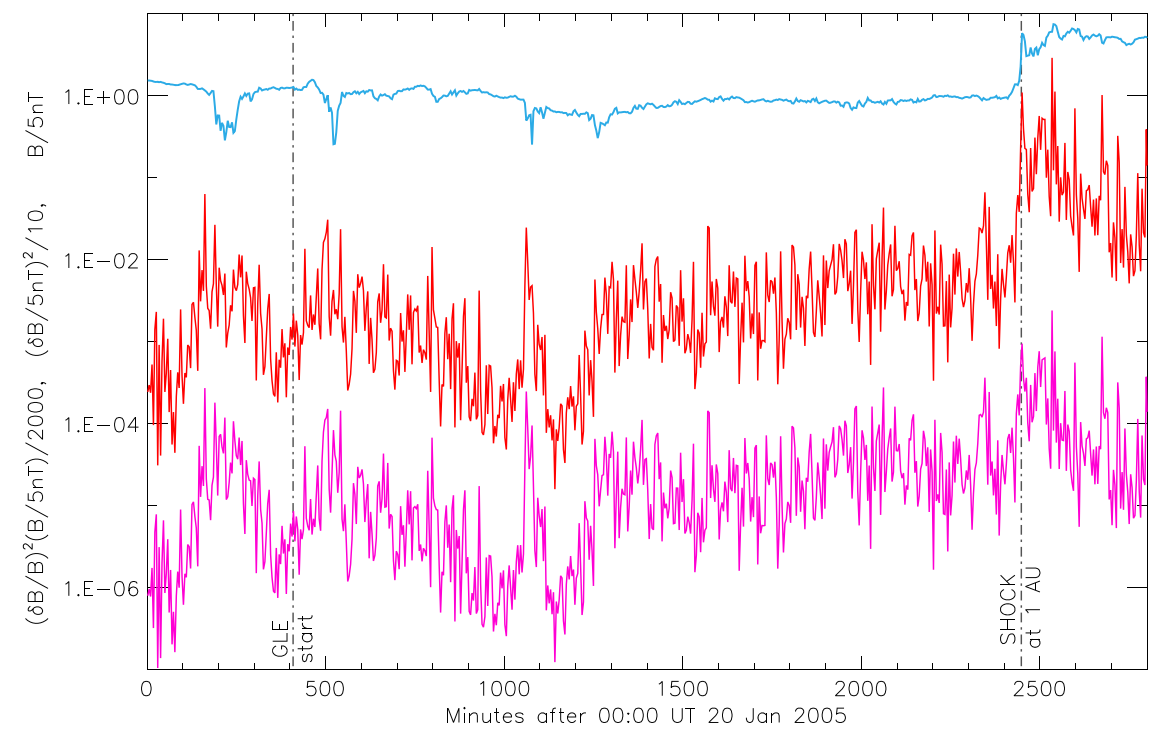

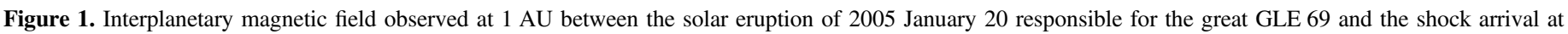

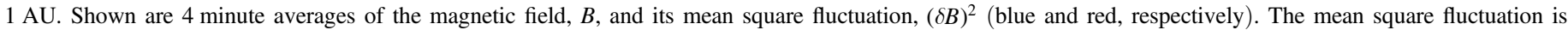

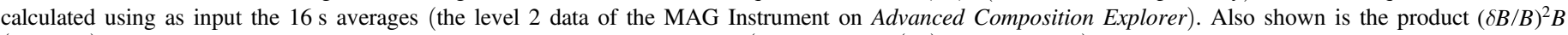
(magenta), which is relevant to the diffusion coefficient of energetic protons (e.g., Equation (14) in Section 3.2).

by the particles streaming from the shock (Bell 1978; Lee 2005; Vainio \& Laitinen 2007; Ng \& Reames 2008). High-energy $(\gtrsim 100 \mathrm{MeV})$ proton spectra observed in solar events are steep, so that there may be not enough particles at high energies to excite the resonant waves. On the other hand, the external turbulence that could assist the high-energy particle acceleration near the Sun would simultaneously prevent the shock-accelerated particles from escaping to 1 AU. A compromise between fast acceleration at the shock and prompt access of the accelerated protons to $1 \mathrm{AU}$ can be achieved in structured solar wind (Kocharov et al. 2013, 2014).

It is proposed that a shock propagates near the Sun in the environment comprising both highly turbulent magnetic tubes and quiet magnetic tubes. There are numerous indications on the flux-tube texture of the solar wind at $1 \mathrm{AU}$ and its effect on the SEP transport (e.g., Mazur et al. 2000; Borovsly 2008; Chollet \& Giacalone 2008, 2011; Qin \& Li 2008). An intermittence of proton scattering conditions was observed in the SEP and GLE event of 1998 May 2 (GLE 56; Kocharov et al. 2007a). Figure 1 shows a pattern of the contrasting level of magnetic fluctuations in the interplanetary magnetic tubes connected to the near-Sun source of high-energy protons in the famous event of 2005 January 20 (GLE 69). It is seen that the turbulence level changes by more than one order of magnitude on the timescale of 200-500 minutes. If the solar wind structure co-rotates with the Sun, such a timescale corresponds to the angular size of $2^{\circ}-5^{\circ}$ in solar longitude. While there are no in situ observations close to the Sun, we assume that a similar intermittence of the turbulence level exists also there. It is also expected that the turbulence is strongly enhanced after the passage of the shock (Morfill \& Scholer 1977; Vainio \& Schlickeiser 1999; Zank et al. 2010). High-energy particles may be accelerated by the CME shock near the Sun mainly in the turbulent tubes and then may escape via a cross-field transport to the neighboring quiet tubes and along them to $1 \mathrm{AU}$.

The propagation of charged particles across the mean field direction has been studied for several decades. Jokipii (1966) derived cross-field diffusion coefficients based on a quasilinear approach and field line random walk (FLRW). The FLRW result, however, disagrees with galactic cosmic-ray observations (Burger et al. 2000) and direct particle simulations (Giacalone \& Jokipii 1999) which give the ratio of the perpendicular and parallel diffusion coefficients $D_{\perp} / D_{\|} \sim 0.01$ near the Earth. More recently, Matthaeus et al. (2003) formulated a nonlinear guiding center theory that compounds the FLRW to take into account the scattering of particles along the magnetic field, resulting in better consistency with the observations. It should be noted that SEP observations in the interplanetary medium suggest $D_{\perp} / D_{\|}$values varying from $\sim 10^{-5}$ inferred from SEP intensity dropouts during impulsive events (e.g., Dröge et al. 2010; Wang et al. 2014) to $D_{\perp} / D_{\|} \sim 0.1$ required to explain multi-spacecraft SEP observations (Dresing et al. 2012; Dröge et al. 2014). Thus, the ratio $D_{\perp} / D_{\|}=0.01-0.1$ may be considered as an optimistic estimate.

Our previous modeling of the shock acceleration in structured solar wind (Kocharov et al. 2013, 2014) was focused on production of SEPs observed in the interplanetary medium and did not consider possible precipitation of accelerated particles back into the solar atmosphere behind the shock. However, the quiet magnetic tubes previously proposed for the SEP escape into the interplanetary medium could facilitate also the particle transport back to the Sun. The fraction of particles returning to the Sun from the CME bow shock is expected to be small because of magnetic line convergence in the sunward direction, but it may be not negligibly small for production of presently observable secondary emissions. Another potentially important process ignored in the previous modeling was stochastic re-acceleration of the shock-accelerated particles by the turbulence amplified by the shock. Both these processes will be considered in the present work. In addition, we will modify the geometry of the system to make the SEP escape cone wider. The latter does not change the underlying physics but may be helpful for the future data interpretation. We take advantage of the strong first-order 
Fermi acceleration at the shock front, the strong second-order acceleration in the downstream region, and the strong longitudinal gradient in wave turbulence, to accelerate and emit particles detected as a GLE and transport particles back to the Sun to radiate as high-energy $\gamma$-rays.

\section{MODEL}

\subsection{The Field-aligned Diffusion-Convection Equation (DCE)}

As the particle mean free path for efficient acceleration in a quasi-parallel shock shall be small, the modeling can be based on the DCE of cosmic-ray transport (Toptygin 1985, Section 8.1 , and references therein):

$$
\frac{\partial M_{a}}{\partial t}=\nabla \cdot \boldsymbol{D} \cdot \nabla M_{a}-\boldsymbol{u} \cdot \nabla M_{a}+\frac{p}{3} \frac{\partial M_{a}}{\partial p} \nabla \cdot \boldsymbol{u},
$$

where $p$ is particle momentum; $\boldsymbol{D}$ is diffusion tensor in the coordinate space $\boldsymbol{r} ; M_{a}=M_{a}(\boldsymbol{r}, p, t)$ is the particle distribution function averaged over pitch angle (the omnidirectional distribution function); $\boldsymbol{u}$ is hydrodynamic velocity of the ambient plasma. However, Equation (1) does not account for possible motion of the particle scattering centers with respect to plasma. If particles are scattered by waves or other irregularities propagating in the local plasma frame in a different direction, the DCE includes also the stochastic acceleration term that accounts for a net effect of the head-on and overtaking collisions between particles and scattering centers (e.g., Melrose 1980; Schlickeiser 1989, 2003, Section 8.3 of the former monograph and Section 12.3.2 of the latter one):

$$
\begin{aligned}
\frac{\partial M_{a}}{\partial t}= & \nabla \cdot \boldsymbol{D} \cdot \nabla M_{a}-\boldsymbol{u} \cdot \nabla M_{a} \\
& +\frac{p}{3} \frac{\partial M_{a}}{\partial p} \nabla \cdot \boldsymbol{u}+\frac{1}{p^{2}} \frac{\partial}{\partial p} p^{2} D_{p} \frac{\partial M_{a}}{\partial p},
\end{aligned}
$$

where the diffusion coefficient in momentum space, $D_{p}$, depends on the velocity distribution of scattering centers in the plasma frame. Note that $\boldsymbol{u}$ should be understood here as the hydrodynamic velocity of scattering centers, and in the case of their anisotropic distribution the velocity $\boldsymbol{u}$ may deviate somewhat from the hydrodynamic velocity of plasma. However, for the sake of simplicity, such correction to $\boldsymbol{u}$ will not be included in the present modeling.

Equation (2) may be rewritten for the particle energy distribution, $F$, instead of the momentum distribution, $M_{a}$, $F(\boldsymbol{r}, E, t)=4 \pi p^{2} M_{a}(\boldsymbol{r}, p, t) d p / d E \quad$ (e.g., Section 8.1 of Toptygin 1985):

$$
\begin{aligned}
\frac{\partial F}{\partial t}= & \nabla \cdot \boldsymbol{D} \cdot \nabla F-\nabla \cdot(\boldsymbol{u} F)+\frac{1}{3} \frac{\partial}{\partial E}(a E F) \nabla \cdot \boldsymbol{u} \\
& -\frac{\partial}{\partial E}\left(\dot{E}_{\mathrm{st}} F\right)+\frac{\partial^{2}}{\partial E^{2}}\left(D_{E} F\right),
\end{aligned}
$$

where $E$ is kinetic energy,

$$
a=\frac{d \ln E}{d \ln p}=\frac{E+2 m c^{2}}{E+m c^{2}},
$$

and momentum diffusion is expressed in terms of systematic energy gain and random walk in energy, with the acceleration rate and the energy diffusion coefficient

$$
\begin{aligned}
\dot{E}_{\mathrm{st}} & =\frac{1}{p^{2}} \frac{\partial}{\partial p} a p E(p) D_{p}(p), \\
D_{E} & =(a E)^{2} \frac{D_{p}(p)}{p^{2}} .
\end{aligned}
$$

The momentum diffusion coefficient, $D_{p}(p)$, is adopted in the standard, quasi-linear form (e.g., Schlickeiser \& Steinacker 1989).

The field-aligned form of the DCE operates with a linear number density of energetic particles, i.e., the number of particles per unit of a magnetic tube length:

$$
N(\boldsymbol{r}(\xi, i), E, t)=\Phi_{i} \frac{F(\boldsymbol{r}, E, t)}{B(\boldsymbol{r}, t)},
$$

where $\xi$ is a curvilinear coordinate along a particular magneticfield tube $i ; \Phi_{i}$ is the magnetic flux of the tube, assumed hereafter to be the same for all tubes (Kocharov et al. 2012). Then we neglect particle drifts and adopt the diffusion coefficient as

$$
D_{\alpha \beta}=D_{\|} b_{\alpha} b_{\beta}+D_{\perp}\left(\delta_{\alpha \beta}-b_{\alpha} b_{\beta}\right),
$$

where $\boldsymbol{b} \equiv \boldsymbol{B} / B, \boldsymbol{B}$ is the magnetic intensity vector, and $\delta_{\alpha \beta}$ is the Kronecker delta. By substituting the product $N B$ instead of the volumetric number density $F$ into Equation (3) and splitting operator $\nabla$ into components parallel and perpendicular to the magnetic field, then using Gauss's law $\nabla \cdot \boldsymbol{B}=0$ and the induction equation $\partial \boldsymbol{B} / \partial t=\nabla \times(\boldsymbol{u} \times \boldsymbol{B})$, after straightforward but rather lengthy manipulation one can recast the DCE into the field-aligned form, in which magnetic field lines serve as coordinate lines:

$$
\begin{aligned}
\frac{\partial N}{\partial t}= & \frac{\partial}{\partial \xi} D_{\|} \frac{\partial N}{\partial \xi}-\frac{\partial}{\partial \xi}\left(u_{\|}+\frac{D_{\|}}{L_{B}}\right) N \\
& +\frac{1}{B} \nabla_{\perp} \cdot D_{\perp} \nabla_{\perp}(N B)-\boldsymbol{u}_{\perp} \cdot \nabla_{\perp} N+N \boldsymbol{u}_{\perp} \cdot \frac{\partial \boldsymbol{b}}{\partial \xi} \\
& +\frac{1}{3} \frac{\partial}{\partial E}(a E N) \nabla \cdot \boldsymbol{u}-\frac{\partial}{\partial E}\left(\dot{E}_{\mathrm{st}} N\right)+\frac{\partial^{2}}{\partial E^{2}}\left(D_{E} N\right),
\end{aligned}
$$

where $u_{\|}$and $\boldsymbol{u}_{\perp}$ are the hydrodynamic velocity's components parallel and perpendicular to the magnetic field, and $L_{B}=-B /(d B / d \xi)$ is magnetic focusing length. The first two terms on the right-hand side of Equation (8) represent particle diffusion and advection along magnetic tubes. The next term describes diffusion in two perpendicular directions (the twodimensional vector operator $\nabla_{\perp} \equiv \nabla-\boldsymbol{b}(\partial / \partial \xi)$ should include Lamé coefficients appropriate to a particular curvilinear coordinate system). The fourth term accounts for the change of particle "populations" due to the magnetic tube change, as different tubes sweep past the fixed point in coordinate space. The fifth term on the right-hand side of Equation (8) is the linear density change of energetic particles caused by the local stretching (or shortening) of the magnetic-field-line element. The last three terms represent the adiabatic acceleration/ deceleration in the converging/diverging plasma flow, the regular energy gain due to stochastic acceleration, and the 


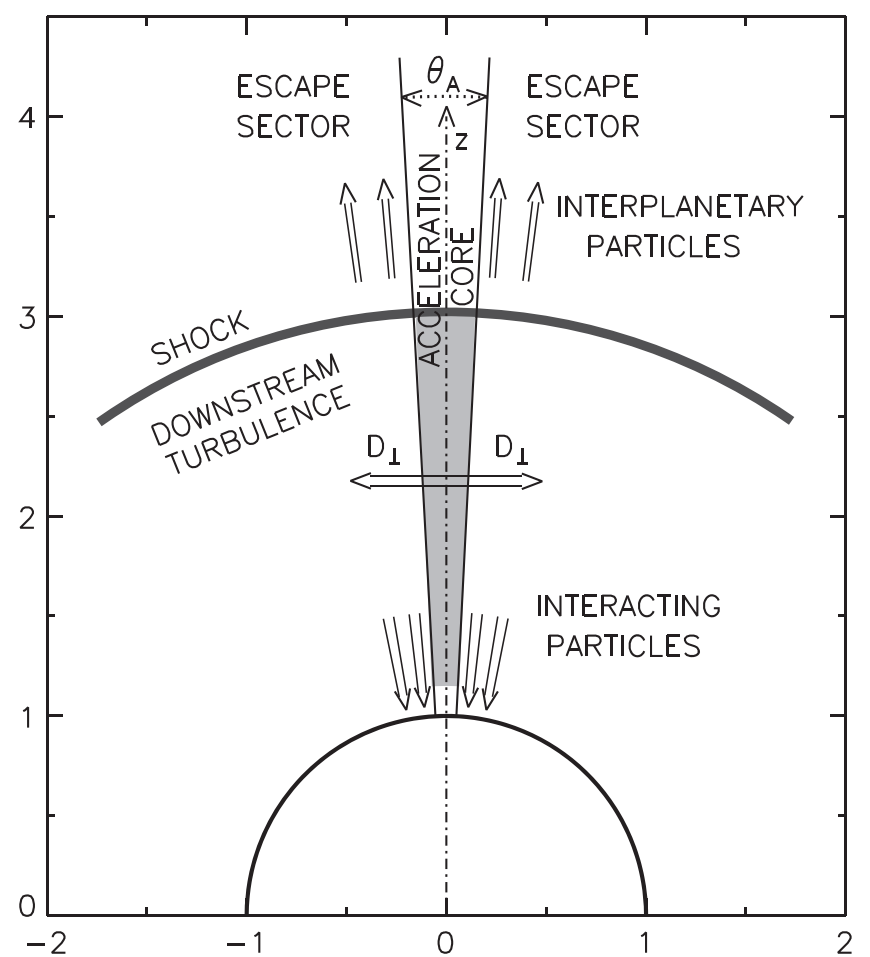

Figure 2. Particle acceleration and transport model. The region of stochastic re-acceleration of the shock-accelerated particles is shaded. The vertical extent of this region depends on the total number of resonant protons.

associated random walk in energy, respectively. The fieldaligned form of the DCE employs the natural coordinate system defined by magnetic field lines and hence is convenient for Monte Carlo modeling of the charged particle acceleration and transport.

\subsection{Monte Carlo Simulation}

We consider a radial magnetic field, $\boldsymbol{B}=B_{\odot}\left(R_{\odot} / r\right)^{2}(\boldsymbol{r} / r)$, and model particle acceleration in the heliocentric spherical shock wave expanding through the structure comprising the highly turbulent conic ray (the acceleration core $\theta_{A}$ in Figure 2) embedded in a wider cone of quiet plasma via which the accelerated particles can escape (the particle escape sector). The system is assumed to possess azimuthal symmetry around the axis $z$. Hence, in the direction perpendicular to the magnetic field, only dependence on the polar angle $\theta$ will be considered. Thus, Equation (8) in the spherical coordinates is reduced to

$$
\begin{aligned}
\frac{\partial N}{\partial t}= & \frac{\partial}{\partial r} D_{\|} \frac{\partial N}{\partial r}-\frac{\partial}{\partial r}\left(u+\frac{D_{\|}}{L_{B}}\right) N \\
& +\frac{1}{r^{2} \sin \theta} \frac{\partial}{\partial \theta} D_{\perp} \sin \theta \frac{\partial N}{\partial \theta}+\frac{1}{3} \frac{\partial}{\partial E}(a E N) \frac{\partial u}{\partial r} \\
& -\frac{\partial}{\partial E}\left(\dot{E}_{\mathrm{st}} N\right)+\frac{\partial^{2}}{\partial E^{2}}\left(D_{E} N\right),
\end{aligned}
$$

where $N(r, \theta, E, t)$ is the particle distribution over the length $r$ of the magnetic tube of solid angle $\Delta \Omega=2 \pi \sin \theta \Delta \theta$; $u=u(r)$ is the hydrodynamic speed of the radial flow associated with the assumed spherical shock; the magnetic focusing length in the radial field is $L_{B}=r / 2$.
In Equation (9), the polar angle $\theta$ can be naturally replaced by the virtual transverse linear coordinate $Y$ defined as

$$
Y=(1-\cos \theta) R_{o}
$$

which is proportional to the solid angle: $d Y=R_{o} d \Omega /(2 \pi)$ where $R_{o}$ is a normalization length. With this substitution, the DCE in the radial magnetic field looks like the equation of twodimensional diffusion on the $r-Y$ plane with coordinatedependent diffusion coefficients:

$$
\begin{aligned}
\frac{\partial N}{\partial t}= & \frac{\partial}{\partial r} D_{\|} \frac{\partial N}{\partial r}+\frac{\partial}{\partial Y} D_{\perp}\left(2-\frac{Y}{R_{o}}\right) \frac{R_{o} Y}{r^{2}} \frac{\partial N}{\partial Y} \\
& -\frac{\partial}{\partial r}\left(u+\frac{2 D_{\|}}{r}\right) N+\frac{1}{3} \frac{\partial}{\partial E}(a E N) \frac{\partial u}{\partial r} \\
& -\frac{\partial}{\partial E}\left(\dot{E}_{\mathrm{st}} N\right)+\frac{\partial^{2}}{\partial E^{2}}\left(D_{E} N\right) .
\end{aligned}
$$

In the perpendicular diffusion term, the coefficient $\left(2-Y / R_{O}\right)$ may be set simply to 2 , if the transverse "linear size" of the system $Y \ll 2 R_{o}$, which implies a possible maximum spread of the considered magnetic tube bundle $2 \theta<75^{\circ}$. Note that the radial advection caused by magnetic focusing, $2 D_{\|} / r$, is strong near the Sun and will present a major obstacle for particle precipitation to the solar chromosphere.

We adopt the normalization length $R_{o}=200 R_{\odot}$ and the angular half-size of the acceleration core $\theta_{A} / 2=0.05 \mathrm{rad}$ $\left(\theta_{A}=5.7\right)$, which implies the core's lateral boundary at $Y_{A} \equiv Y\left(\theta_{A} / 2\right)=0.25 R_{\odot}$. The outer boundary of the escape sector is placed at $4 Y_{A}$ (i.e., at $\theta=0.1 \mathrm{rad}$ ), which for the goals of the present paper is not very important. Thus, the simulation box in the $r-Y$ plane will be a rectangle: $0 \leqslant Y \leqslant R_{\odot}$ and $R_{\odot} \leqslant r \leqslant 21 R_{\odot}$. The lateral boundary $Y=0$ (inner boundary) corresponds to the acceleration core axis (symmetry axis of the system). Both $Y$-boundaries of the simulation box will be treated as closed, while both $r$-boundaries will be open, providing the particles escape toward or away from the Sun. Note that the simulation box is artificially bounded at the heliocentric angle $\theta=5.7$ and thus not aimed at modeling a GLE's actual extent over the heliolongitude.

Low-frequency Alfvén waves, well below the proton cyclotron frequency, are easily generated and weakly decaying waves (e.g., Kaplan \& Tsytovich 1973, Section 1.7), which can accelerate super-Alfvénic protons via the gyroresonance interaction. High levels of the Alfvén wave turbulence were observed or proposed in different coronal and solar wind structures (e.g., Miller 1991a, 1991b; van der Holst et al. 2014, and references therein). In particular, Alfvén waves were identified in the solar wind (after Neugebauer et al. 1978) and near the interplanetary shock waves ( $\mathrm{Hu}$ et al. 2013). For these reasons, the last two terms of Equation (11) will be ascribed to the stochastic acceleration by the Alfvén wave turbulence.

The model takes into account interaction of protons with Alfvén waves in the quasi-linear approximation, in which particles can interact only with resonant waves. The resonance condition for energetic protons is (Jokipii 1966)

$$
k v \mu= \pm \omega_{B} / \gamma
$$

where $k$ is the wavenumber, $v, \mu$, and $\gamma$ are velocity, pitchangle cosine, and Lorentz factor of the particle, $\omega_{B}$ is its 
cyclotron frequency, and the sign depends on the wave polarization. The gyroresonant interaction causes the proton scattering and acceleration. The corresponding particle diffusion coefficient parallel to the magnetic field is of the form (e.g., Section 9.3 of Toptygin 1985; Schlickeiser \& Steinacker 1989):

$$
D_{\|}(E, \boldsymbol{r}, t)=D_{o}(\boldsymbol{r}, t) p^{3-q} \gamma^{-1},
$$

where $q$ is the spectral index of the wave turbulence. Hereafter the Kraichnan turbulence spectrum, $q=1.5$, will be employed. A similar spectrum was reported for the solar wind in the frequency range $0.0001-1 \mathrm{~Hz}$ (e.g., Section 3.3 of Toptygin 1985; Vörös 2014). However, the turbulence spectrum index is not a crucial parameter of the model, so the Kolmogorov spectrum, $q=\frac{5}{3}$, could be equally used. The quasi-linear mean free path corresponding to Equation (13) can provide a reasonable approximation for protons in a wide energy range (Bieber et al. 1994). The energy density of the waves resonant with non-relativistic protons is linked to the model parameter $D_{\|}$by the estimate (e.g., Toptygin 1985):

$$
w\left(k>\omega_{B} / v\right)=\left[\frac{(\delta B)^{2}}{4 \pi}\right]_{k>\omega_{B} / v} \approx \frac{B^{2}}{8 \pi} \frac{v^{2}}{\omega_{B} D_{\|}(E)},
$$

where $B$ is the regular magnetic field, $B=B_{\odot}\left(R_{\odot} / r\right)^{2}$.

We model the spherical shock (Figure 2) as a narrow continual compression propagating in the radial direction. For present simulations, the shock speed is $u_{\mathrm{sh}}=2100 \mathrm{~km} \mathrm{~s}^{-1}$, the upstream plasma $\beta=0.3$, the Alfvénic Mach number $M_{A}=3$, and the corresponding gas compression ratio in the shock $\sigma=3.64$. In the solar frame, the plasma is motionless upstream of the shock and moves outwards after the shock arrival. The hydrodynamic velocity steeply increases inside the shock front up to the final value prescribed by the Rankine-Hugoniot jump condition. We assume a linear decrease of the hydrodynamic velocity behind the shock, so that the velocity returns to zero at the solar surface. This implies additional adiabatic deceleration of particles behind the shock.

Due to the turbulence amplification by the shock, the parallel diffusion coefficient decreases in the shock front from the upstream value $D_{\| 1}$ to $D_{\| 2} \ll D_{\| 1}$ downstream of the shock. We adopt $D_{\| 2}=D_{\| 1} / 20$ (Section 18.3 of Toptygin 1985; Vainio \& Schlickeiser 1999). We propose that the diffusion coefficient changes also in the direction perpendicular to the magnetic field at the transition from the acceleration core to the escape sector (halo):

$$
D_{\|}(Y)=\frac{1}{2}\left[D_{\| A}+D_{\| B}-\left(D_{\| A}-D_{\| B}\right) \tanh \left(\frac{Y-Y_{A}}{\Delta_{Y}}\right)\right],
$$

where indexes $A$ and $B$ correspond to core and halo, respectively. We adopt the parallel diffusion coefficient of a $0.1 \mathrm{MeV}$ proton upstream of the shock $D_{\| 1 A}(0.1 \mathrm{MeV})=$ $2 \times 10^{6} \mathrm{~km}^{2} \mathrm{~s}^{-1}$, the turbulence energy jump between core and halo $w_{A} / w_{B}=D_{\| B} / D_{\| A}=50$ (cf. Figure 1), and the interface width $\Delta_{Y}=0.1 R_{\odot}$. The perpendicular diffusion coefficient is a fixed fraction of the parallel one: $D_{\perp}=0.04 D_{\|}$.

After crossing the shock, Alfvén waves change both their overall energy and the relative amount of waves propagating in opposite directions. The latter is characterized by the turbulence cross-helicity $H_{c}$, which is the difference in the intensities of forward- and backward-propagating waves as a fraction of the total intensity (e.g., Dung \& Schlickeiser 1990). The cross-helicity is important for stochastic re-acceleration because only waves propagating in mutually opposite directions can cause the acceleration described by the last two terms of Equation (11). The energy diffusion coefficient $D_{E}$ and the energy gain rate $\dot{E}_{\text {st }}$ are connected by Equations (5) and may be parameterized as

$$
\begin{aligned}
& D_{E}=(a E)^{2} \frac{\alpha_{2} v_{\mathrm{A} 2}^{2}}{3 D_{\| 2}}, \\
& \dot{E}_{\mathrm{st}}=\frac{a E}{p^{3}} \frac{\partial}{\partial E} \frac{p^{3}}{a E} D_{E},
\end{aligned}
$$

where $v_{\mathrm{A} 2}$ is the downstream value of Alfvén speed and $\alpha_{2}$ is the turbulence form factor. The form factor $\alpha$ is estimated as the product of the spectral factor $\alpha_{s}=3 /\left[q(4-q)\left(4-q^{2}\right)\right]$ of isotropic turbulence (Schlickeiser \& Steinacker 1989) and the fractional abundance of the equal-intensity waves propagating in forward and backward directions $\alpha_{c}=1-\left|H_{\mathrm{c}}\right|$ (fraction of "isotropic" turbulence): $\alpha=\alpha_{s} \alpha_{c}$. Thus, we estimate the turbulence form factor for stochastic acceleration downstream of the shock as

$$
\alpha_{2}=\frac{3\left(1-\left|H_{\mathrm{c} 2}\right|\right)}{q(4-q)\left(4-q^{2}\right)} .
$$

In isotropic turbulence $\alpha=\alpha_{s}$, and it would be 0.46 at spectral index $q=1.5$ or 0.63 at $q=\frac{5}{3}$. However, the shock-amplified turbulence is not expected to be isotropic, which results in a smaller value of the parameter $\alpha_{2}$. Transmission of Alfvén waves through the parallel shock wave was calculated by Vainio \& Schlickeiser (1999). The resulting downstream crosshelicity state depends on the shock speed, the upstream plasma $\beta$, and the upstream cross-helicity $H_{c 1}$. In particular, if there are no forward-propagating (in our case, sunward-propagating) waves upstream of the shock, then for the shock compression ratio $\sigma<3.3$ the cross helicity $H_{c 2}$ is very close to -1 and hence $\alpha_{2} \approx 0$. In the case of "isotropic" upstream turbulence $\left(H_{c 1}=0\right)$ and $\beta=0.3$, the downstream cross-helicity $H_{c 2}$ is from -0.8 to -0.6 for shock compression ratios from 3.5 to 3.8 (as can be inferred from Figures 3 and 4 of Vainio \& Schlickeiser 1999) and hence $\alpha_{2}=0.09-0.18$ at $q=1.5$. Hereafter, similar to Kocharov et al. (2011) we adopt $\alpha_{2}=0.15$. The case of $\alpha_{2}=0$ (no stochastic re-acceleration) will also be considered. Stochastic acceleration upstream of the shock is neglected in both cases, because of the low turbulence level there.

In major SEP events the re-accelerated protons may consume a significant fraction of the turbulence energy and thus the wave-particle interaction should be treated self-consistently (for a recent example see Afanasiev et al. 2014). For the goals 
of the present research, such self-consistent treatment is largely simplified. The pitch-angle dependence in the resonance condition is left out, so that hereafter we will consider the resonant waves only with the wavenumber $k=\omega_{B} /(\gamma v)$. Then the wave energy distribution over the wavenumber, $d w / d k \sim k^{-q}$, is replaced with the wave energy distribution over the resonant proton energy $E\left(v \gamma=\omega_{B} / k\right)$, which can be cast as

$$
\frac{d w}{d E}=\frac{\omega_{B}}{m v^{3} \gamma^{1 / 2}} \frac{d w}{d k}
$$

where $m$ is the proton mass. In the non-relativistic limit, for the Kraichnan phenomenology upstream of the shock this suggests the incoming turbulence spectrum $d w / d E \propto E^{-0.75}$. In the course of re-acceleration the protons gain their energy from the energy of Alfvén waves, and energy conservation between the particles and the waves should be obeyed.

Our present model secures only the time-integrated balance of the energy exchange between protons and waves with a simplified approach. Particles under the re-acceleration take their energy from the turbulence left after the shock, and consequently, the turbulence has to decay with distance from the shock. We assume that the post-shock excess of the turbulence energy density decays exponentially behind the shock: $\Delta w \sim\left(D_{\|}^{-1}-D_{\| 1}^{-1}\right) \sim \exp [X / \rho]$, where $\rho$ is the decay length and $X \equiv\left(r-r_{\text {shock }}\right)<0$, and hence

$$
\frac{1}{D_{\|}(X)}=\frac{1}{D_{\| 1}}+\left(\frac{1}{D_{\| 2}}-\frac{1}{D_{\| 1}}\right) e^{X / \rho} .
$$

The turbulence decay length $\rho$ depends on the number of particles with energy $E$ available in the magnetic tube $Y$ : $\rho=\rho(E, Y)$. All other dependencies of the wave distribution are ignored. The function $\rho(E, Y)$ is adjusted using an iterative procedure to meet the energy conservation between particles and waves integrated over the simulated time interval.

We assume that $D_{\| 1}$ does not depend on the distance from the Sun at $r<0.1$ AU. In such a case the turbulence energy density $(\delta B)^{2} /(4 \pi) \propto B \propto r^{-2}$ (Equation (14)) and hence the turbulence energy per unit of the magnetic tube length upstream of the shock,

$$
\left[\frac{d w\left(k>\omega_{B} / v\right)}{d r}\right]_{1}=\left[\frac{(\delta B)^{2}}{4 \pi}\right]_{k>\omega_{B} / v} \Delta \Omega r^{2},
$$

is constant; here $\Delta \Omega$ is the solid angle of the radial magnetic tube; $E<100 \mathrm{MeV}$. For the waves in resonance with protons of energy $<E$, the waves' total energy pumped up behind the shock over the shock transit from $r=R_{\min }$ to $R_{\max }$ can be estimated as

$$
W(<E)=\left[\frac{d w\left(k>\omega_{B} / v\right)}{d r}\right]_{1} \frac{D_{\| 1}}{D \|_{2}} \frac{R_{\max }-R_{\min }}{\sigma} .
$$

Using Equation (14), the total energy $W(<E)$ can be expressed in terms of the upstream diffusion coefficient. Because of the energy conservation, stochastic re-acceleration of the shockaccelerated protons cannot raise their total energy by more than $W(<E)$.
Seed protons for the shock acceleration are picked up in front of the shock from an exponential energy distribution with mean energy $\langle E\rangle_{\text {seed }}=300 \mathrm{keV}$ with uniform volumetric number density along a fixed magnetic field line. It is assumed, however, that the number density of seed particles in different magnetic tubes is different, being proportional to the turbulence energy density in the tube, i.e., $N_{\text {seed }}(Y) \propto 1 / D_{\| 1}(Y)$.

The field-aligned DCE (Equation (11)) is solved with a standard method of stochastic simulations of the random walk and advection of Monte Carlo particles (e.g., Fletcher 1994) in two spatial coordinates and energy. In particular, stochastic reacceleration is simulated by changing at each Monte Carlo time step $\delta t$ the particle energy by a small value

$$
(\delta E)_{\mathrm{st}}=\dot{E}_{\mathrm{st}} \delta t+R_{\mathrm{G}} \sqrt{2 D_{E} \delta t},
$$

where $R_{\mathrm{G}}$ is a random number picked from the standard Gaussian distribution. Such a process results in advection and diffusion in energy identical to those described by the two last terms of Equation (11), even though real processes of the wave-particle interaction are not explicitly simulated. We trace the energy of each Monte Carlo particle and simultaneously accumulate the total energy gained by the particles of energy $E$ from their resonant waves, to reach finally the energy conservation between particles and waves.

The shock is launched nominally at the solar surface $(t=0$, $\left.r=R_{\odot}\right)$ but the particle acceleration starts only upon the shock arrival at $r=R_{\min }=1.5 R_{\odot}$ and continues up to $R_{\max }=6.4 R_{\odot}$ ( $t=30$ minutes). At both $r$-boundaries, the particle number density is set to zero by fast escapes. At both $Y$-boundaries, particles are returned to the simulation box. Accelerated particles are registered at each Monte Carlo time step to collect the particle distributions inside the acceleration region. Particles are also registered upon their escape at the top of the simulation box $\left(r=21 R_{\odot}\right)$ and at the bottom of the box $\left(r=R_{\odot}\right)$ to accumulate the distributions of interplanetary particles and interacting particles, respectively.

\section{RESULTS}

Two modeling cases are considered. In case A, stochastic reacceleration is turned off by setting the parameter $\alpha_{2}=0$ in Equation (16). In case B, we assume significant re-acceleration by adopting $\alpha_{2}=0.15$. All other model parameters are identical. Figure 3 maps the time-integrated spatial distributions of accelerated protons in the shock frame for three energy channels, in $\mathrm{MeV}$, decaMeV, and hectoMeV ranges. Particles are accelerated by the shock mainly in the highly turbulent core at $Y<0.15 R_{\odot}$, then diffuse in the direction perpendicular to the magnetic field (along $Y$ ) and escape to the interplanetary space or return to the Sun along magnetic field lines of the escape sector at $Y>0.25 R_{\odot}$. It is seen that stochastic reacceleration increases the number of energetic protons and shifts the distribution maximum from the shock to its downstream region. Attenuation of the downstream turbulence due to the stochastic re-acceleration facilitates the cross-field transport of $\mathrm{MeV}$ protons in case $\mathrm{B}$ (top right panel of Figure 3).

Figure 4 shows the time-average volume-integrated energy spectra of protons inside the simulation box (internal spectra) with or without stochastic re-acceleration (thick solid curve and dashed curve, respectively) along with time-integrated spectra of protons escaping into the interplanetary medium or 

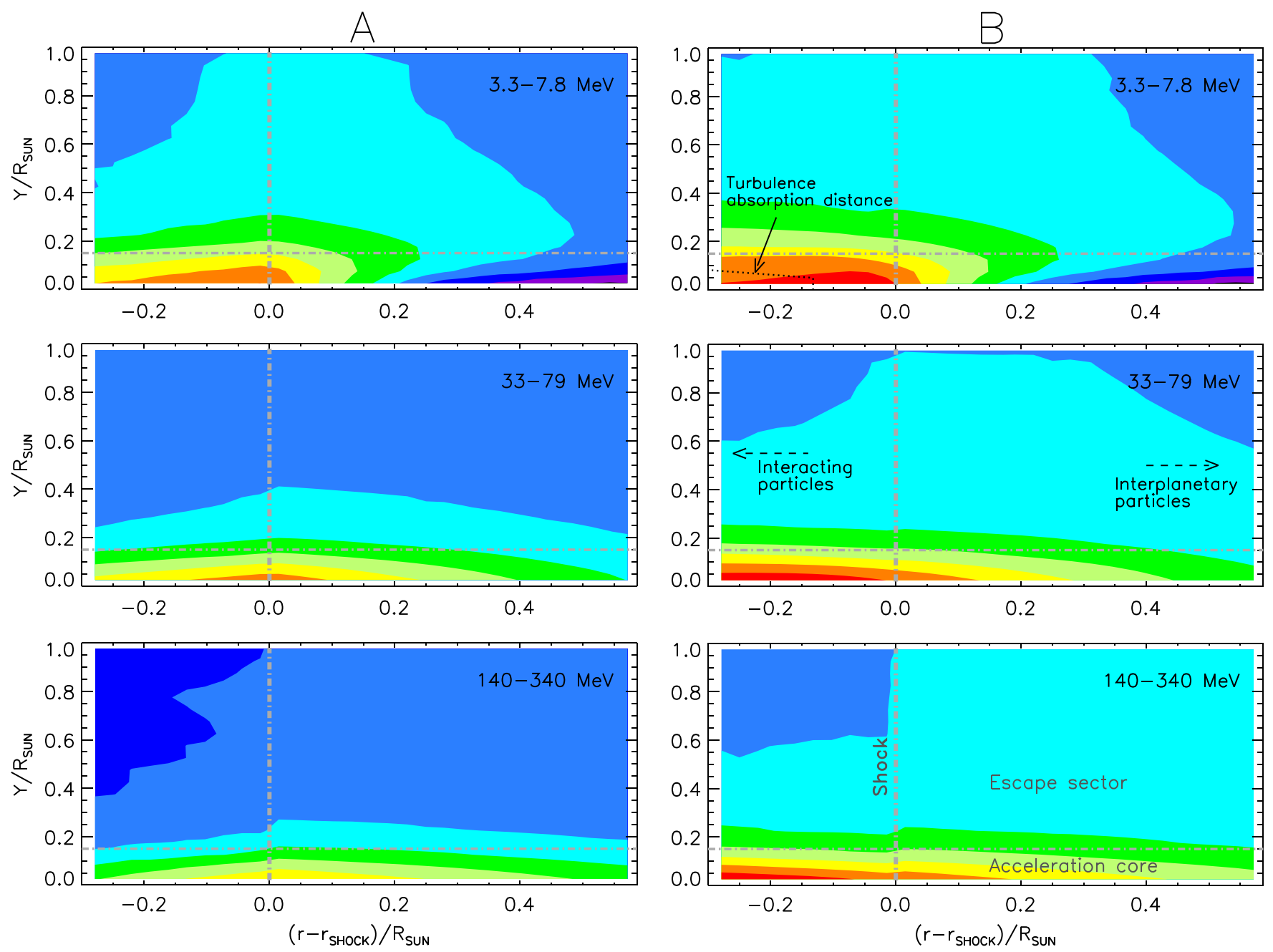

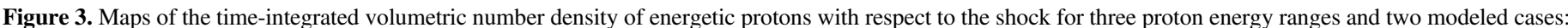

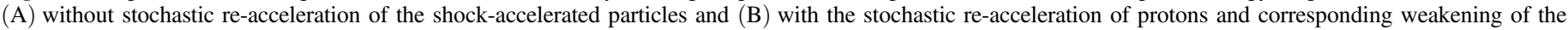

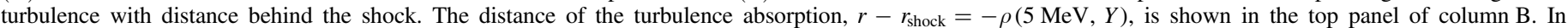

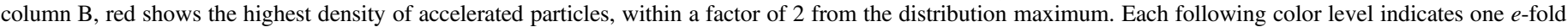

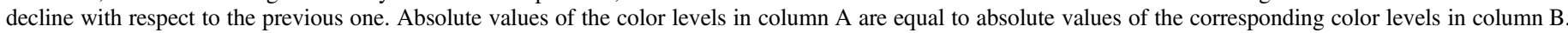
The boundary between the acceleration core and the escape sector is placed at $Y=Y_{A}-\Delta_{Y}$.

precipitating into the solar chromosphere (points). Thin solid curves are identical to the thick solid curve but shifted down for comparison with other spectra. The internal spectra are of a power-law shape, typical for shock acceleration, with a highenergy rollover caused by the limited acceleration time and particle escape across the magnetic field. Stochastic reacceleration affects both parts of the shock-accelerated spectrum: a stronger effect is seen above the rollover, while the low-energy part of the spectrum retains a power law shape with slightly altered slope (uppermost thin solid curve versus dashed curve). The escaping particle spectrum at low energies strongly depends on the assumed energy dependence of the perpendicular diffusion coefficient. In the considered case of $D_{\perp} \propto D_{\|} \propto E^{0.75}$, the escaping spectra are harder than the corresponding internal spectra. However, the difference is less significant in the hectoMeV range (points versus two lower thin solid curves). The strongest effect of stochastic reacceleration is seen in protons precipitating to the Sun (red closed points of case B versus open points of case A).

Figure 5 shows the downstream turbulence-energy distribution over the resonant proton energy, $W(E)$, in different magnetic tubes (curves 1-4), which is the total turbulence energy excess supplied behind the shock in the selected magnetic tubes over the shock transit from $R_{\min }$ to $R_{\max }$. The energy dependence $W(E)$ is according to Equation (19), the $Y$ dependence is $W(Y) \sim 1 / D(Y)$ with $D(Y)$ given by Equation (15), while the normalization comes from Equations (22), (21), and (14). Consistently with this normalization, the turbulence energy in Figure 5 is in units of

$$
A=8 \times 10^{31} \frac{B_{\odot}}{1 \mathrm{Gs}}
$$

(the function $W$ is dimensionless). Not more than the total wave energy $W(E, Y)$ can be consumed by the protons of energy $E$ in the tube $Y$ in the course of stochastic reacceleration. Points in Figure 5 show the simulated energy gain of protons due to stochastic acceleration. In the acceleration core (series 1 ) the decaMeV protons absorb the entire excess of turbulence behind the shock, while only a fraction of the turbulence energy is consumed by protons of higher energies or outside the acceleration core. Consistently with Figure 5, the 


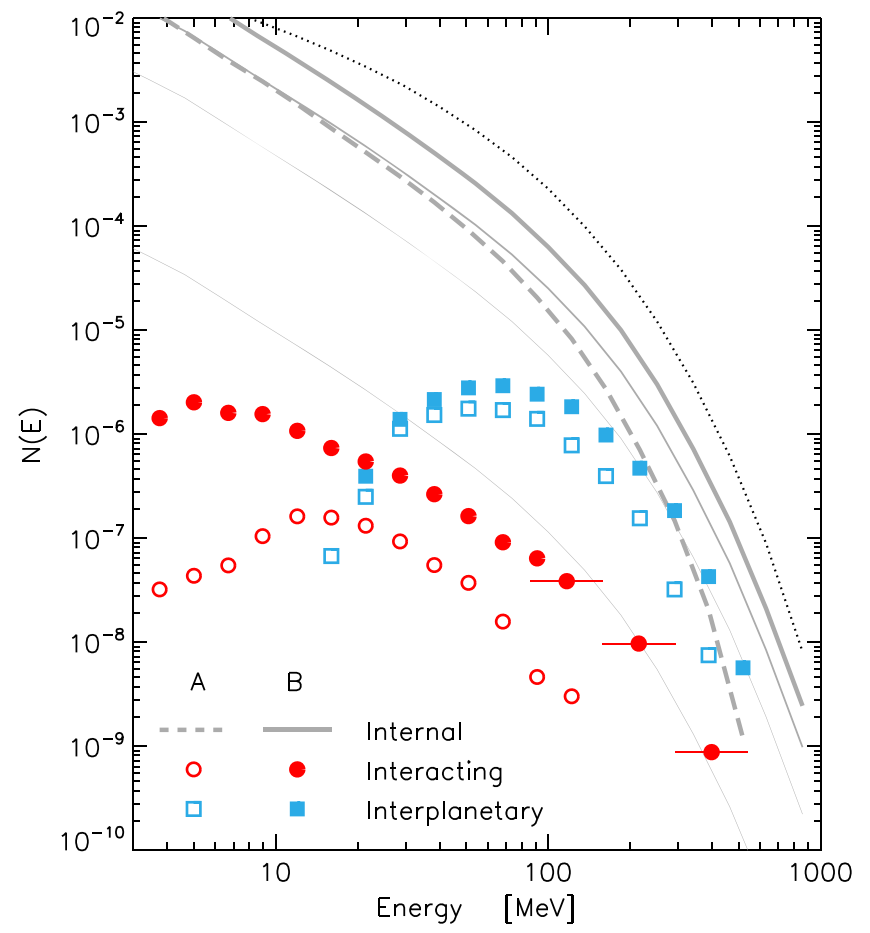

Figure 4. Energy spectra of accelerated protons for the two modeled cases: (A) without stochastic re-acceleration of the shock-accelerated particles and (B) with stochastic re-acceleration. The internal distribution is the time-average volume-integrated distribution inside the simulation box. Interacting and interplanetary distributions are the time-integrated distributions of particles escaping at the bottom and the top of the simulation box, respectively. For easy comparison betweens the cases A and B, and between the escaping and internal spectra of case B, the internal distribution of case B (heavy solid curve) is shown also with altered normalization (three light solid curves). The dotted line additionally shows the internal spectrum that would be attained in the test particle regime, where the effect of accelerated particles on waves is neglected. The integration time is 30 minutes.

proton spectra of Figure 4 are in the units of $A$ particles per $\mathrm{MeV}$. Note that the total number of high-energy protons in our modeling is comparable to what may be estimated from the observations of the SEP and GLE event of 1998 May 2 (Figure 3(c) of Kocharov et al. 2007b).

The self-consistent treatment of particles and waves makes the particle spectrum after stochastic acceleration a kind of power law in the energy range where the entire turbulence enhancement has been absorbed. This would not be expected at very small density of seed particles (in the test particle regime - dotted curve in Figure 4). The proton spectrum inside the acceleration region (internal spectrum) is shifted in energy after the re-acceleration by a factor of 1.75 (thick solid curve versus dashed curve), i.e., almost half the energy of accelerated protons comes from the turbulence.

Figure 6 shows the ratio of integral energy spectra of particles precipitating to the solar chromosphere and particles escaping into the interplanetary medium. It is seen that stochastic re-acceleration can significantly increase the interacting-to-escaping particle ratio, which still stays small. Time profiles of the particle emission into the interplanetary space and precipitation into the chromosphere are shown in Figure 7. Injection of interacting protons continues at a nearly constant rate to beyond the simulation period of 30 minutes, with significantly higher intensity in the case of stochastic reacceleration behind the shock.

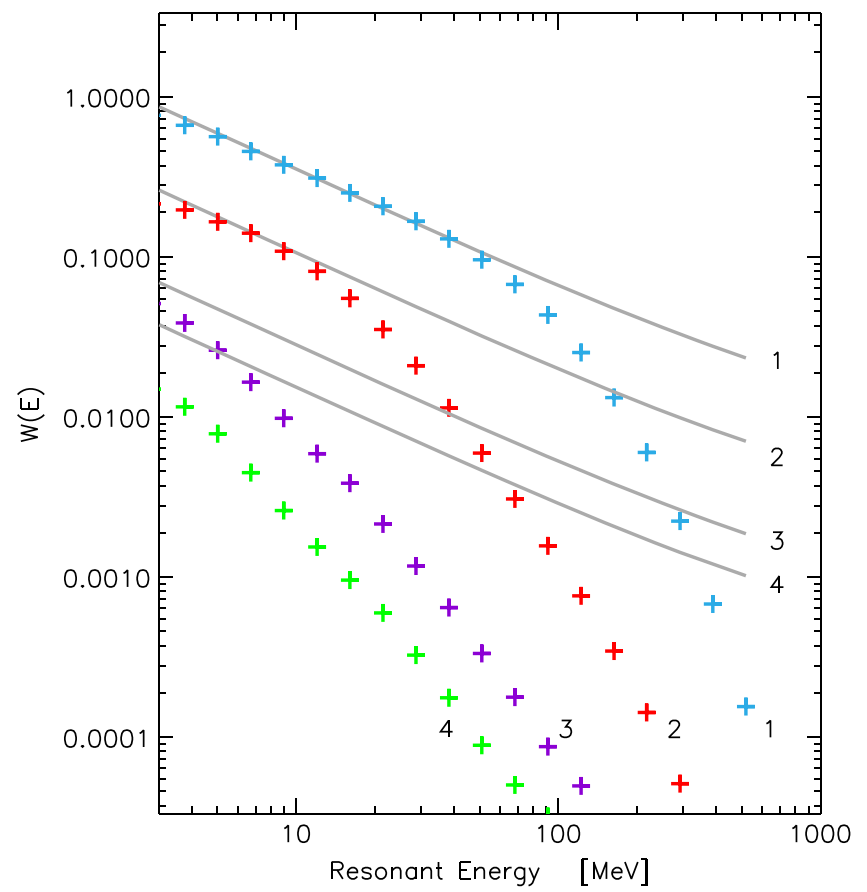

Figure 5. Enhancement of turbulence energy spectrum behind the shock at different tangential distances from the system axis: $(1) Y=(0-0.1) R_{\odot},(2)$ $Y=(0.1-0.2) R_{\odot}$, (3) $Y=(0.2-0.3) R_{\odot}$, and (4) $Y=(0.3-0.4) R_{\odot}$, integrated over the simulated time period and plotted as a function of the resonant proton energy, $W(E)$ (gray curves). Points show the turbulence energy absorbed by protons during the stochastic re-acceleration downstream of the shock in the corresponding magnetic tubes $1-4$. It is seen in particular that inside the acceleration core (tube 1) the turbulence energy enhancement is completely absorbed by protons of energy up to $60 \mathrm{MeV}$.

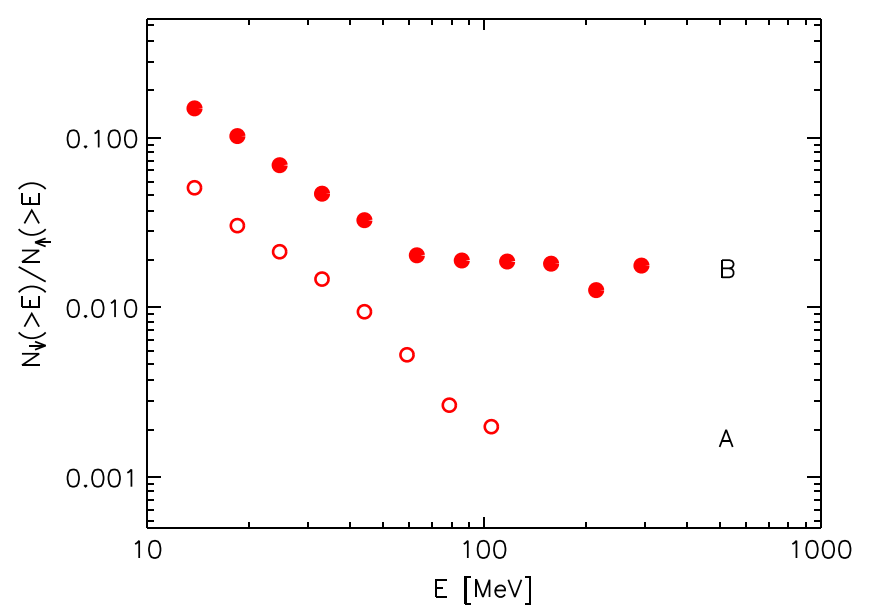

Figure 6. Number ratio of interacting protons to interplanetary protons with energy $\geqslant E$ for the model cases $\mathrm{A}$ and $\mathrm{B}$ (irregularities are due to statistical limitations).

\section{DISCUSSION}

There were two previous theoretical calculations of the ratio of numbers of interplanetary to interacting particles: for the stochastic acceleration on open magnetic field lines in the solar corona (Kocharov et al. 1999) and for the diffusive shock acceleration (Vainio et al. 2000). It was shown in the first paper that divergence of the coronal magnetic field lines results in a few times larger number of particles emitted into the interplanetary space as compared to particles injected for nuclear interaction in the solar chromosphere. Seed particles for 


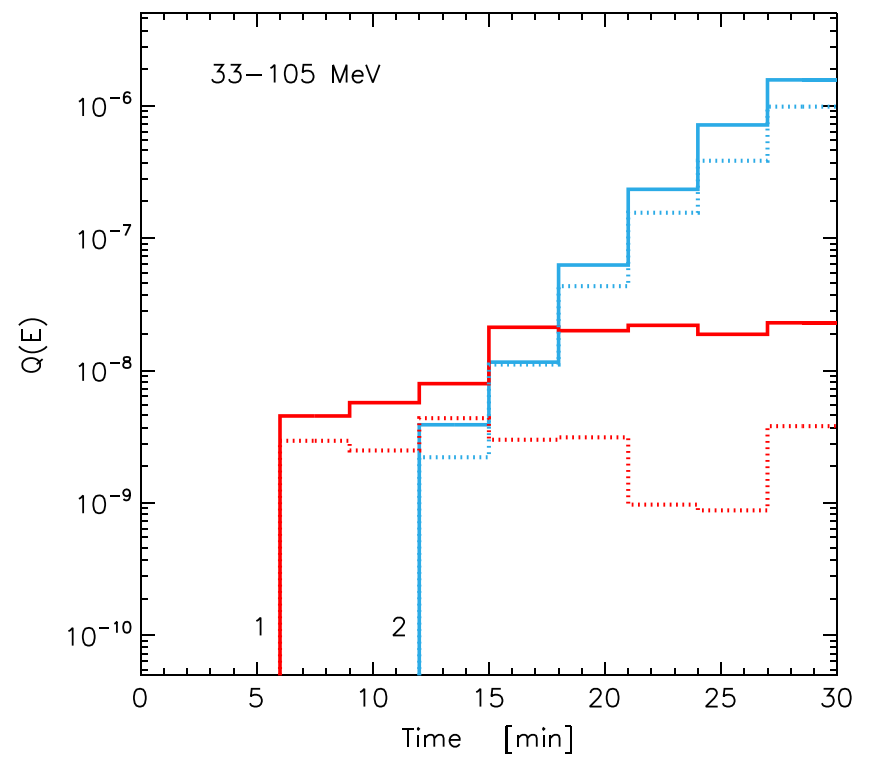

Figure 7. Simulated time profiles of the 33-105 MeV proton precipitation rate to the Sun $(1-$ red) and the proton escape rate to the interplanetary medium (2-blue) for the model cases A (dotted) and B (solid).

the stochastic acceleration were assumed to be of such low energy and low number density that they could not affect the resulting high-energy particle spectrum, which consequently was the Bessel-type spectrum typical for stochastic acceleration of test particles (e.g., Equation (23) of Kocharov et al. 1999). In contrast, now we start stochastic acceleration with particles already accelerated in the shock and our present treatment of stochastic acceleration is self-consistent, that is the acceleration duration depends on the available amount of resonant waves. This makes the final spectrum of accelerated particles a power law in the energy range where the entire amount of wave energy can be absorbed by the accelerated particles (Figures 4 and 5). A qualitatively similar result has been recently obtained by Afanasiev et al. (2014) using a more rigorous, dynamic selfconsistent approach. In that paper, however, we did not simulate the shock acceleration but assumed an initial power law proton spectrum below $30 \mathrm{MeV}$ to be stochastically reaccelerated in a closed box (e.g., in a magnetic loop). Another previous modeling of interplanetary protons versus interacting protons was for the acceleration in a parallel shock without stochastic re-acceleration (Vainio et al. 2000). However, particle transport between the shock and the chromosphere was not properly considered. In the present model, the problem of particle transport from the departing shock back to the chromosphere is solved with the assumption of the fast transport sector and the cross-field transport from the acceleration core to the transport sector.

Sufficiently fast cross-field transport of SEPs downstream of the shock is an essential ingredient of our model. The crossfield transport can be caused by meandering of field lines, due to turbulent fluctuations, and the particle decoupling from the meandering field lines. Recently Laitinen et al. (2013) showed that the large cross-field extent of SEP events can be explained by their initial non-diffusive propagation along meandering field lines, which also provides an explanation for the intensity dropouts, while retaining the time-asymptotic diffusive behavior caused by the decoupling. In the case of particle propagation in the shock upstream, due to the large parallel diffusion coefficient the particles may reach long distances from the shock along meandering field lines before decoupling. In the turbulent shock downstream, however, the meandering may not be the primary factor contributing to the widening of the SEP population across the magnetic field, as the particles may decouple faster from their field lines. The current timeasymptotic diffusion descriptions based on meandering of field lines are likely unable to describe cross-field transport in such a configuration. Thus, in this work we model the transport parameters simply by keeping the ratio of cross-field and parallel diffusion coefficients constant throughout the simulation box.

The energy dependence of cross-field transport, $D_{\perp}(E)$, is ruled by its nature and is essential for both interplanetary particles and interacting particles. The model energy spectrum of escaping protons would be close to their spectrum inside the simulation box if the cross-field transport was independent of energy (similar to Kocharov et al. 2013, Figure 4 therein). The $D_{\perp} / D_{\|}$ratio may depend on the particle velocity and the turbulence energy level (Zank et al. 2004; Li et al. 2012, and references therein). Shalchi et al. (2009) have considered a compound diffusion of charged particles in the solar wind at the high turbulence level, taking into account particle drift, advection and wave propagation effects. Their results indicate that perpendicular mean free path may increase as the proton energy decreases below $\sim 300 \mathrm{MeV}$ (their Figure 1). Such behavior, if applied in our model, would lift up the low-energy part of the spectrum of escaping particles, making it qualitatively similar to the power-law spectrum produced inside the acceleration core.

In addition to the meandering of field lines and particle decoupling from the field lines, the changing of the magnetic connectivity in the downstream region may also play a role in cross-field propagation of particles. The plasma $\beta$ in the model shock changes from $\beta<1$ to $\beta>1$, and a possible effect of magnetic reconnections to the particle propagation cannot be ruled out, especially if the acceleration core includes opposite magnetic polarities, which perhaps could also assist with generation of waves and seed particles upstream of the shock.

Diffusive shock acceleration requires frequent scattering along the magnetic field. The scattering may be provided by the turbulence generated by the accelerated particles themselves, instead of the pre-existing external turbulence considered in our present model. The present modeling, however, is focused on the high-energy part of the SEP spectrum where the number of particles is small and may not be sufficient for the wave generation. While we neglect the effect of proton-amplified waves, the validity of this assumption is tested in a post-hoc manner. Protons accelerated by the shock can overtake it for about one diffusion length $L_{D}=D_{\| 1} / u_{\text {sh }}$ and maintain there the proton flux anisotropy $a_{1} \approx u_{\mathrm{sh}} / v$. Anisotropy of the proton distribution may cause amplification of the resonant Alfvén waves (e.g., Section 11.2.1.2 of Schlickeiser 2003). The energy transferred from a proton to the resonant Alfvén wave in a single collision is $d E= \pm v_{A} \mid d p_{\|}$, because the scattering is elastic in the wave frame. Over the small time $\delta t$, a proton may transfer to resonant waves the energy $\delta E \approx v_{A} p v \delta t / \lambda$. However, oppositely moving protons can absorb the waves' energy, so that the wave growth is contributed only by the anisotropic part of the proton distribution, $a_{1} n(E)$. After the shock transit time over the diffusion length, $\Delta t=D_{\| 1} / u_{\mathrm{sh}}^{2}$, the total energy transferred from protons to waves in the unit 
volume is $\Delta E \approx a_{1} n(E) v_{A} p v \Delta t / \lambda$. This will not exceed the external turbulence energy density, $w_{1}(E)$, if the energy density of accelerated protons at the shock is relatively small:

$$
E n(E) \leqslant M_{A} w_{1}(E),
$$

We check the accelerated proton density against this threshold by calculating the parameter

$$
\Gamma(E, Y)=\frac{E n(E)}{M_{A} w_{1}(E)} \approx \frac{E N_{\mathrm{sh}}(E, Y)\left(R_{\max }-R_{\min }\right) D_{\| 1}}{M_{A} W(E, Y) \sigma D_{\| 2}},
$$

where $N_{\mathrm{sh}}(E, Y)$ is the time-average linear number density of accelerated protons near the shock and $W(E, Y)$ is the total wave-energy supplied downstream of the shock. Self-generated waves can be neglected when the parameter $\Gamma$ is small. In the modeling case $\mathrm{B}$, the contribution of self-generated waves may be noticeable only at low energies in the middle of the acceleration core, where $\Gamma$ reaches $1-1.1$ at $E=3-10 \mathrm{MeV}$, while it is below 0.5 outside the core. In the hectoMeV range, the contribution of self-generated waves is small everywhere: $\Gamma<0.1$.

The modeling results essentially depend on the assumed distribution of seed particles and turbulence upstream of the shock. The basic assumption of the model is that both the seed particles and the turbulence are concentrated in a narrow region called the acceleration core. The model parameter value $\alpha_{2}=0.15$ assumed in case B implies that a significant amount of sunward-propagating waves exists in the acceleration core before the arrival of the shock. Such waves should be locally generated together with seed particles, e.g., due to magnetic reconfiguration before the main CME. This may be relevant to the observed GLE production in the aftermath of previous CMEs (Aschwanden 2012).

The time profile of the SEP emission into the interplanetary space (Figure 7) depends on the assumed distribution of seed particles with distance from the Sun. We adopt a volumetrically uniform distribution, which actually implies an increasing number of seed particles per unit of heliocentric solid angle as the shock travels farther from the Sun. If the seed particle injection was terminated, e.g., at $4 R_{\odot}$, the high-energy proton emission into the interplanetary space would reach its maximum value within the simulated time interval, and the interacting-to-interplanetary proton ratio would be a few times higher than that shown in Figure 6. This and other model parameters should be further adjusted after a detailed comparison with observational data.

Some previous case studies of the SEP and GLE events indicate that there may be more than one high-energy ion component contributing to interplanetary and interacting particles in a single event (e.g., Kocharov et al. 1996, 2012). Careful case studies are required to reveal, perhaps among others, the interacting particle component produced by the CME-driven shocks on open magnetic field lines.

\section{CONCLUSION}

We have considered a hypothesis that solar hectoMeV-to$\mathrm{GeV}$ ions are accelerated in a narrow radial ray where both suprathermal seed particles and turbulence are available before the CME shock arrives and from where SEPs can escape via cross-field transport. We have studied the simplest model of this kind and conclude the following.

1. Ions can be accelerated to several $\mathrm{GeV}$ with a realistically steep spectrum at above $100 \mathrm{MeV}$ and promptly emitted into the interplanetary space or back to the Sun.

2. The total number of interplanetary ions exceeds by an order of magnitude the number of protons producing the high-energy secondary emissions via nuclear interactions at the Sun.

3. Stochastic re-acceleration of the shock-accelerated ions is significant, especially for the interacting ions, while the shape of the particle energy spectrum after the reaccelerations may be qualitatively similar to the original shock spectrum.

4. The simultaneous availability of seed particles and turbulence in a narrow region within a few solar radii from the Sun prior to the shock may be crucial for producing a GLE.

5. Efficient cross-field transport of the accelerated ions should also be provided downstream of the shock and its properties are important.

In future research we hope to compare these theoretical findings and ideas with solar multi-wavelength observations and the high-energy ion detection at the Earth's orbit.

One of authors (L. K.) thanks Dr. Gerald Share for inspiring discussion at the 2013 AGU Fall Meeting and comments. This research was supported by the UK Science and Technology Facilities Council (grant ST/J001341/1) and by the Academy of Finland through projects 260596 and 267186 . We thank the ACE MAG instrument team and the ACE Science Center for providing the ACE data.

\section{REFERENCES}

Ackermann, M., Ajello, M., Albert, A., et al. 2014, ApJ, 787, 15

Afanasiev, A., Vainio, R., \& Kocharov, L. 2014, ApJ, 790, 36

Ajello, M., et al. 2014, ApJ, 789, 20

Aschwanden, M. A. 2012, SSRv, 171, 3

Axford, W. I., Leer, E., \& Skadron, G. 1977, in Proc. 15th Int. Cosmic Ray Conf. 11 (Sofia: B'lgarska Akademiia na Naukite), 132

Bell, A. R. 1978, MNRAS, 182, 147

Bieber, J. W., et al. 1994, ApJ, 420, 294

Blandford, R. D., \& Ostriker, J. P. 1978, ApJL, 221, L29

Borovsly, J. E. 2008, JGRA, 113, A08110

Burger, R. A., Potgieter, M. S., \& Heber, B. 2000, JGRA, 105, 27447

Chollet, E. E., \& Giacalone, J. 2008, ApJ, 688, 1368

Chollet, E. E., \& Giacalone, J. 2011, ApJ, 728, 64

Debrunner, H., Lockwood, J. A., \& Ryan, J. M. 1993, ApJ, 409, 822

Dresing, N., Gómez-Herrero, R., Klassen, A., et al. 2012, SoPh, 281, 281

Dröge, W., Kartavykh, Y. Y., Dresing, N., Heber, B., \& Klassen, A. 2014, JGRA, 119, 6074

Dröge, W., Kartavykh, Y. Y., Klecker, B., \& Kovaltsov, G. A. 2010, ApJ, 709, 912

Dung, R., \& Schlickeiser, R. 1990, A\&A, 237, 504

Fletcher, L. 1994, SSRv, 68, 139

Giacalone, J., \& Jokipii, J. R. 1999, ApJ, 520, 204

Hu, Q., Zank, G. P., Li, G., \& Ao, X. 2013, in AIP Conf. Proc. 1539, Proc. 13th Int. Solar Wind Conf. (New York: AIP), 175

Hua, X.-M., \& Lingenfelter, R. E. 1987, SoPh, 107, 351

Jokipii, J. R. 1966, ApJ, 146, 480

Kahler, S. 1994, ApJ, 428, 837

Kaplan, S. A., \& Tsytovich, V. N. 1973, Plasma Astrophysics, Vol. 59 (Oxford: Pergamon)

Kocharov, L., Cho, K.-S., \& Valtonen, E. 2011, ApJ, 735, 4

Kocharov, L., Kovaltsov, G. A., Laitinen, T., Mäkela, P., \& Torsti, J. 1999, ApJ, 521, 898

Kocharov, L., Laitinen, T., \& Vainio, R. 2013, ApJL, 778, L5 
Kocharov, L., Laitinen, T., Usoskin, I., \& Vainio, R. 2014, ApJL, 787, L21

Kocharov, L., Torsti, J., Vainio, R., Kovaltsov, G. A., \& Usoskin, I. G. 1996 $\mathrm{SoPh}, 169,181$

Kocharov, L., Saloniemi, O., Torsti, J., Kovaltsov, G., \& Riihonen, E. 2007a, ApJ, 654, 1121

Kocharov, L., Saloniemi, O., Torsti, J., et al. 2007b, ApJ, 659, 780

Kocharov, L., Vainio, R., Pomoell, J., et al. 2012, ApJ, 753, 87

Krymsky, G. F. 1977, Dokl. Akad. Nauk SSSR, 234, 1306

Laitinen, T., Dalla, S., \& Marsh, M. S. 2013, ApJL, 773, L29

Lee, M. A. 2005, ApJS, 158, 38

Lee, M. A., Mewaldt, R. A., \& Giacalone, J. 2012, SSRv, 173, 247

Lee, M. A., \& Ryan, J. M. 1986, ApJ, 303, 829

Li, G., Shalchi, A., Ao, X., Zank, G., \& Verkhoglyadova, O. P. 2012, AdSpR, 49, 1067

Lin, R. P. 2005, AdSpR, 35, 1857

Matthaeus, W. H., Qin, G., Bieber, J. W., \& Zank, G. P. 2003, ApJL, 590, L53

Mazur, J. E., Mason, G. M., Dwyer, J. R., et al. 2000, ApJL, 532, L79

McCracken, K. G., Moraal, H., \& Stoker, P. H. 2008, JGRA, 113, A12101

Melrose, D. B. 1980, Plasma Astrophysics, Vol. 2 (New York: Gordon and Breach)

Mewaldt, R. A., Looper, M. D., Cohen, C. M. S., et al. 2012, SSRv, 171, 97

Miller, J. A. 1991a, ApJ, 376, 342

Miller, J. A. 1991b, JGRA, 102, 14631
Morfill, G. E., \& Scholer, M. 1977, Ap\&SS, 46, 73

Murphy, R. J., \& Ramaty, R. 1984, AdSpR, 4, 127

Neugebauer, M., Wu, C. S., \& Huba, J. D. 1978, JGRA, 83, 1027

Ng, C. K., \& Reames, D. V. 2008, ApJL, 686, L123

Qin, G., \& Li, G. 2008, ApJL, 682, L129

Ramaty, R., Mandzhavidze, N., Kozlovsky, B., \& Skibo, J. G. 1993, AdSpR, 13,275

Schlickeiser, R. 1989, ApJ, 336, 243

Schlickeiser, R. 2003, Cosmic Ray Astrophysics (Berlin: Springer)

Schlickeiser, R., \& Steinacker, J. 1989, SoPh, 122, 29

Shalchi, A., Webb, G. M., le Roux, J. A., \& Zank, G. P. 2009, Ap\&SS, 321, 197

Toptygin, I. N. 1985, Cosmic Rays in Interplanetary Magnetic Fields, Vol. 27 (Dordrecht, Moscow: D. Reidel, Nauka)

Vainio, R., \& Schlickeiser, R. 1999, A\&A, 343, 303

Vainio, R., Kocharov, L., \& Laitinen, T. 2000, ApJ, 528, 1015

Vainio, R., \& Laitinen, T. 2007, ApJ, 658, 622

van der Holst, B., et al. 2014, ApJ, 782, 81

Vörös, Z. 2014, ApJL, 797, L10

Wang, Y., Qin, G., Zhang, M., \& Dalla, S. 2014, ApJ, 789, 157

Zank, G. P., Li, G., Florinski, V., et al. 2004, JGRA, 107, A04107

Zank, G. P., Kryukov, I. A., Pogorelov, N. V., \& Shaikh, D. 2010, in AIP Conf. Proc. 1216, Proc. 12th Int. Solar Wind Conf. (New York: AIP), 563 